\title{
Observación del tránsito de Venus desde el Observatorio Astronómico Centroamericano de Suyapa - 5 de junio de 2012
}

Ricardo Antonio Pastrana Sánchez

\section{Resumen}

El 5 de junio de 2012 ocurrió el fenómeno astronómico conocido como el Tránsito de Venus que despertó gran interés a nivel mundial y sobre todo en Honduras ya que no se contaba con registro alguno sobre una observación de este fenómeno. El Departamento de Astronomía y Astrofísica (DAAF) consiente que la vinculación con la sociedad es una responsabilidad que abarca a toda la institución universitaria. Organizó una comisión multidisciplinaria al interior de la Universidad Nacional Autónoma de Honduras (UNAH) encargada de planificar y ejecutar una estrategia de vinculación de la universidad con la sociedad hondureña alrededor del evento científico. Aunque no se cuantifica la cantidad de personas a las que se les informó antes, durante y después del acaecimiento del fenómeno, una evaluación cualitativa del trabajo desarrollado indica que fue un éxito el proceso de vinculación logrado.

Palabras clave: Tránsito de Venus, Vinculación, OACS, Observación.

\section{Abstract}

On June 5, 2012 occurred the astronomical phenomenon known as the Venus Transit which aroused great interest worldwide and especially in Honduras and had not any record about an observation of this phenomenon. The Department of Astronomy and Astrophysics (DAAF) agrees that the relationship with society is a responsibility that encompasses all the university. Organized a multidisciplinary committee within the National Autonomous University of Honduras (UNAH) responsible for planning and executing a strategy of linking the university with Honduran society about scientific event. Although not quantified the amount of people who were informed before, 
during and after the occurrence of the phenomenon, a qualitative assessment of the work undertaken was successful indicates that the process of linking achieved.

Keywords: Venus transit, relationship, OACS, obsevation.

Ricardo Antonio Pastrana Sánchez (r_pastrana@hotmail.com) Universidad Nacional Autónoma de Honduras. Facultad de Ciencias Espaciales. Departamento de Astronomía y Astrofísica. 


\section{Introducción}

El Tránsito de Venus es un evento astronómico que ocurre cuando se alinean el Sol, Venus y la Tierra. El planeta Venus está delante del Sol a la mínima distancia de la Tierra a lo que se le denomina conjunción inferior y durante algunas horas Venus realiza un desplazamiento sobre el disco solar (figura 1) haciéndose notar como un punto oscuro sobre el fondo brillante de la superficie del Sol.

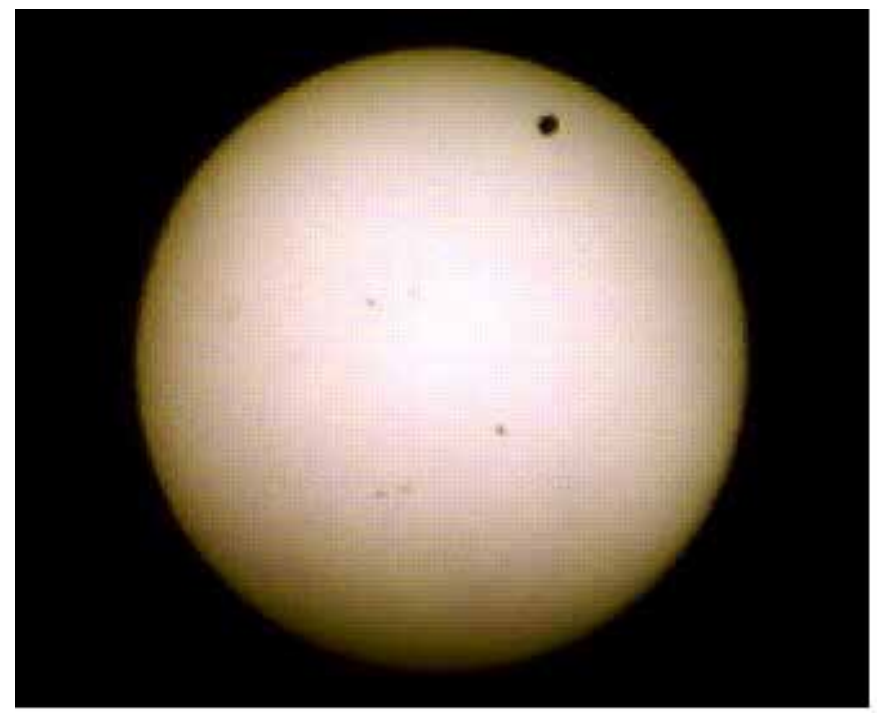

Figura 1. Tránsito de Venus, OACS/UNAH, 5 de junio de 2012.

El fenómeno ha sido de gran importancia para la astronomía. Edmund Halley fue el primero establecer un método usando el tránsito de Venus para calcular la distancia de la Tierra al Sol (Lu y Li 2013) lo que permitió estimar la distancia a otros planetas logrando tener una idea acerca del tamaño de nuestro sistema solar. En la actualidad el método del tránsito es usado para la detección de planetas extrasolares el cual ha sido muy efectivo para la detección de planetas que presentan una órbita cercana a su estrella (Daassou et al, 2010) llamados júpiteres calientes.

Las órbitas de la Tierra y Venus están inclinadas aproximadamente $4^{\circ}$ una respecto a la otra. Esto hace que la frecuencia con que ocurre un tránsito de Venus es muy baja, apenas acontecen dos cada ocho años separados por 125.5 y 105.5 años entre pares consecutivos. 


\section{Importancia de los fenómenos para Honduras}

Los periodos largos en los que se repite esta rara alineación hacen que el evento sea muy llamativo para la sociedad. En Honduras esto fue un caso aún más excepcional ya que el tránsito de venus de 2004 no se pudo apreciar desde nuestra latitud, puesto que aconteció durante la noche del 7 y el amanecer del 8 de agosto. Por otro lado no se cuenta con ningún tipo de registro si alguna población antigua 0 contemporánea haya observado este evento astronómico, ni siquiera los Mayas que su conocimiento de la astronomía se remonta al año 3391 a.c (Smiley 1960). Esto significa que los hondureños por primera vez tendríamos la oportunidad de observar este fenómeno.

Es importante resaltar que el OACS por ser un observatorio de carácter profesional, no solamente se daría a la tarea de obtener imágenes del tránsito que una vez procesadas se convierten en datos cientíicos, sino, además la de aportar conocimiento a la humanidad.

La responsabilidad de ajustar una campaña masiva de informar correctamente por parte del departamento de Astronomía y Astrofísica (DAAF) se vuelve aún mayor considerando que Honduras es un país con una tasa de analfabetismo que es elevada y llega al $15.2 \%$ de la población mayor de 15 años de edad (Banco Centroamericano de Integración Económica 2011). Esto contribuye para que la población fácilmente crea en designios maléficos asociándolos a los fenómenos astronómicos.

\section{Planificación de la estrategia de vinculación}

Parte del logro de una planificación está en su elaboración, la cual se debe hacer con suficiente tiempo para evitar improvisaciones durante su ejecución. El DAAF organizó a su interior una comisión (Tabla 1) con un mes de anticipación a la que se nombró Comisión Tránsito de Venus (CTV) encargada tanto de la observación del evento como del aspecto de vinculación de la UNAH con la sociedad.

\begin{tabular}{|l|l|}
\hline \multicolumn{1}{|c|}{ Cargo } & \multicolumn{1}{|c|}{ Responsable } \\
\hline Coordinador & Ricardo Pastrana \\
\hline Colaboradores & $\begin{array}{l}\text { Norman Palma, Edward Milla, Adán Artola, Paolo Estrada, Yvelice Castillo, José } \\
\text { Jacobo Gámez, Alfredo Gómez, Joel Alemán Maria Quiroz. }\end{array}$ \\
\hline
\end{tabular}

Tabla 1. Comisión Tránsito de Venus. 
Otro punto importante en el cumplimiento de la planificación radica en una evaluación permanente, es decir, las tareas propuestas en el plan de actividades se revisaron conforme a un calendario. Durante las sesiones de evaluación que se desarrollaban semanalmente, los responsables presentaban informe verbal y prueba física de que las obligaciones asignadas se habían realizado o que se estaban ejecutando.

La CTV junto con la Dirección de Vinculación Universidad Sociedad (DVUS) elaboró un cronograma de actividades, el cual contemplaría las tareas observacionales y de vinculación (tabla 2), el trabajo planificado permitió identificar a otras unidades de carácter técnico de la universidad necesarias para el cumplimiento del cronograma. Se invitó a participar a estas unidades con el propósito de formar una comisión institucional (tabla 3) en la donde las unidades participantes son a la vez responsables de aspectos puntuales necesarios para el cumplimiento de la planificación. 


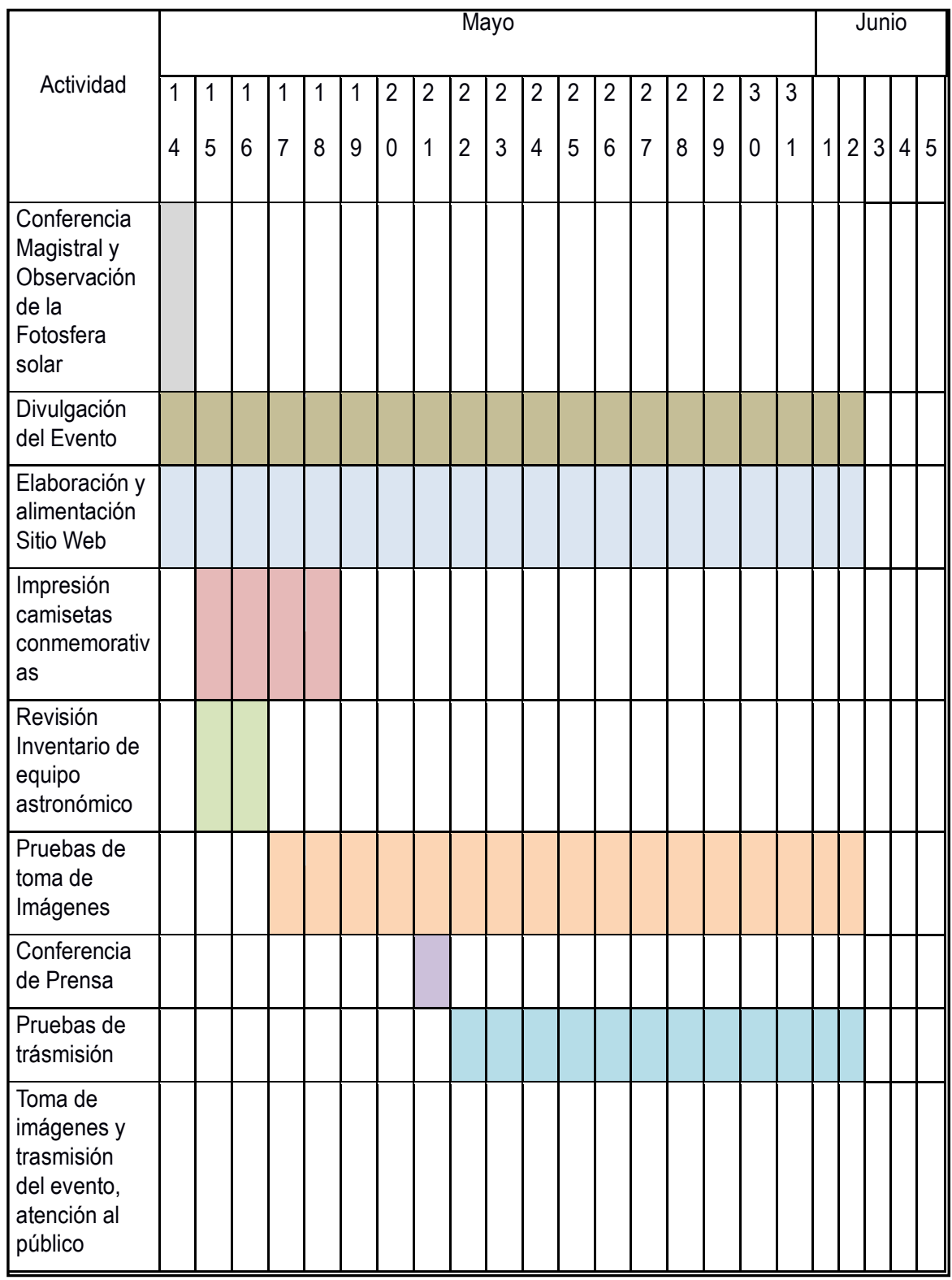

Tabla 2. Cronograma de actividades, Tránsito de Venus DAAF/DVUS 


\begin{tabular}{|l|l|}
\hline \multicolumn{1}{|c|}{ Actividad } & \multicolumn{1}{c|}{ Unidad Responsable } \\
\hline Organizar conferencia de prensa & Vice-Rectoria Académica (VRA) \\
\hline $\begin{array}{l}\text { Asesoria técnica durante la planificación, } \\
\text { programación y seguimiento de las tareas } \\
\text { programadas. }\end{array}$ & $\begin{array}{l}\text { Secretaria Ejecutiva de Desarrollo Institucional } \\
\text { (SEDI) }\end{array}$ \\
\hline Coordinación & Facultad de Ciencias Espaciales. (FACES) \\
\hline Lograr espacios de divulgación & $\begin{array}{l}\text { Dirección de Vinculación Universidad Sociedad } \\
\text { (DVUS) }\end{array}$ \\
\hline Internet de banda ancha & $\begin{array}{l}\text { Dirección Ejecutiva de Gestión de Tecnología } \\
\text { (DEGT) }\end{array}$ \\
\hline Producción y divulgación de videos cortos & Televisión UNAH (UTV) \\
\hline
\end{tabular}

Tabla 3. Comisión institucional.

\section{Actividades realizadas}

La información brindada al público, se hizo llevando a cabo una estrategia de comunicación que iba de lo local a lo nacional y que podemos resumir de la manera siguiente:

Informar a nuestros estudiantes de la asignatura de Introducción a la Astronomía distribuidos en nueve secciones, que abarcaban una población de unas 225 personas en promedio y que además se encontraban en un horario disperso entre las 9:00 y 16:00 horas permitió que gran parte de los universitarios se enteraran acerca del fenómeno.

Se elaboró un boletín informativo de edición especial (figura 2) el cual informaba acerca del porqué sucede este hecho y de su rara frecuencia, de su importancia en el campo de la astronomía y astrofísica, de las zonas en que se podría observar y además de la forma correcta de hacerlo. El boletín fue enviado a los medios de comunicación y además se contó con copias impresas para distribuirlas entre las personas que solicitaban información en el OACS. 


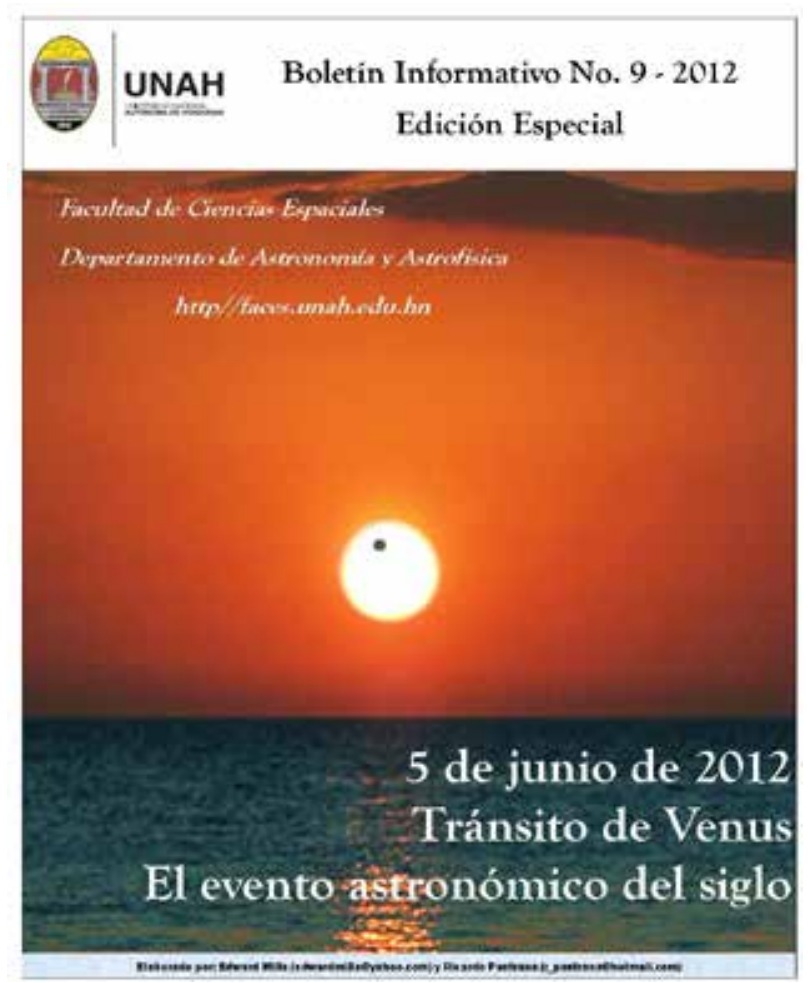

Figura 2. Portada boletín informativo, Tránsito de Venus.

El día lunes 14 de mayo de 2012, de las 14:00 a las 16:00 horas la FACES y del DAAF desarrollaron una conferencia magistral dictada por el Máster en astronomía Norman Palma (figura 3) dirigida a la comunidad universitaria. La finalidad de la actividad fue informar acerca del fenómeno acaecido, además se dispuso instalar un telescopio Celestrón C-8 Schmidt-Cassegrain con una F(mm) de 2032 equipado con un filtro solar (figura 4) para que los asistentes tuvieran la oportunidad de observar la fotosfera solar. Esta actividad fue realizada en Auditorio Central "Juan Lindo" de la UNAH. 


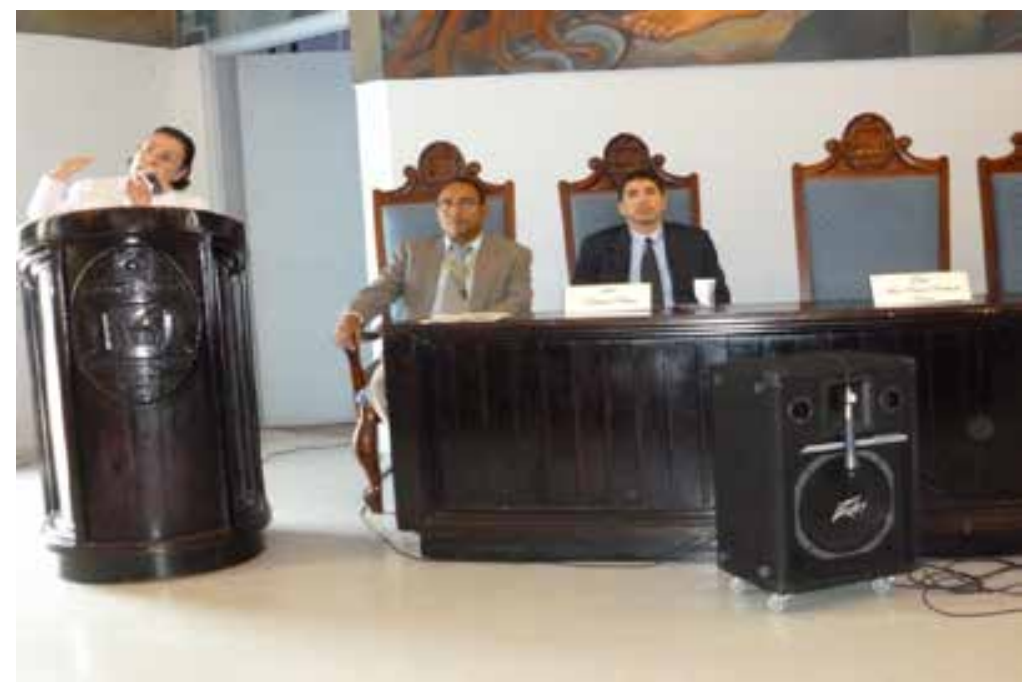

Figura 3. Conferencia magistral Auditorio Central. Izq. a der. Dra. María Cristina Pineda de Carías Decana de la FACES brindando una reseña histórica del Tránsito de Venus, el Lic. Ricardo Pastrana catedrático de astronomía actuando como moderador, Msc. Norman Palma encargado de la conferencia central.

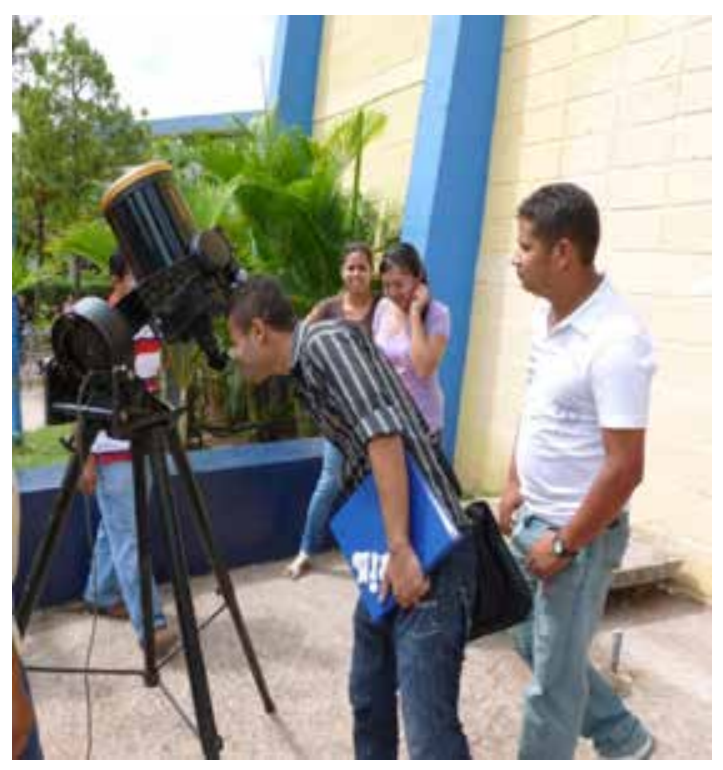

Figura 4. Observación de la fotósfera solar. 
El desarrollo y alimentación de un sitio web http://faces.unah.edu.hn/transitovenus (figura 5) posibilitó que hondureños que no podían visitar el observatorio astronómico, ya sea por la distancia u horario de sus trabajos se informaran de manera correcta sobre el Tránsito de Venus en primer lugar y en segundo lugar tuvieran la oportunidad de seguir mediante el internet la observación del suceso astronómico.

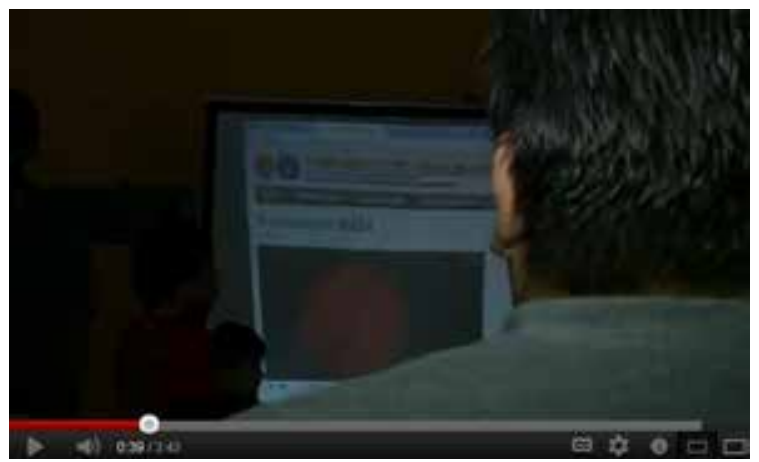

Figura 5. Reportaje programa "Mañana Mix" Museo del niño, San Pedro Sula mientras se seguía el Tránsito de Venus a tráves del sitio web del OACS.

Se imprimieron camisetas conmemorativas al evento (figura 6) y que fueron usadas con anterioridad por el personal docente del DAAF contribuyó por una parte a motivar a la personas sobre la proximidad del acontecimiento astronómico del siglo, y durante la observación los visitantes podían identificar fácilmente a los astrónomos profesionales.

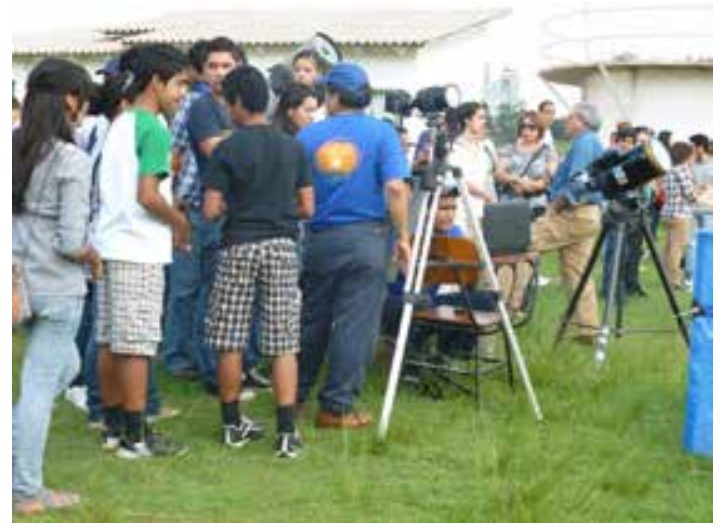

Figura 6. Ing. Edward Milla orientando a los visitantes acerca de la observación fenómeno. 
La UNAH por medio de la Vicerrectoría de Académica, convocó el día martes 29 de mayo a las 10:00 horas a todos los medios de comunicación radiales, escritos y televisivos a una conferencia de prensa (figura 7). La actividad tuvo lugar en las instalaciones de la FACES y estuvieron a cargo sus autoridades la señora decana, la jefa del DAAF y el coordinador de postgrados. Esta acción informativa proporcionó a los hondureños información científica sobre el acontecimiento celestial, la importancia del mismo y cómo observarlo de manera segura.

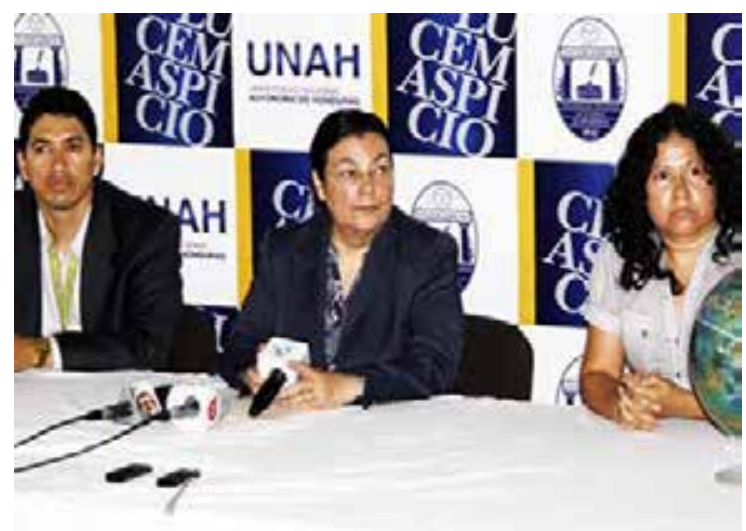

Figura 7. Conferencia de prensa, de izq. a der. Msc. Norman Palma, Dra. María Cristina Pineda Decana FACES y Msc. María Quíroz Jefa DAAF.

La producción de tres videos cortos (figura 8) estuvo a cargo del canal de televisión de la UNAH (UTV), los cuales fueron divulgados por las redes sociales y el canal oficial de la universidad. Estos pueden ser visto en las siguientes direcciones electrónicas: http://www.youtube.com/watch?v=il-_9GfdmN4, http://www. youtube.com/watch?v=B5e_5hw0uwo y http://www.youtube.com/watch?v=BidBgzALaXE.

También cabe destacar la información que fue divulgada por medios digitales, redes sociales y la enorme cantidad de correos electrónicos que circularon informando sobre la observación del tránsito de Venus. Además las muchas entrevistas que se hicieron antes y durante el evento, así como la atención a una gran cantidad de público que asistió al OACS (figura 9), por lo que se dispuso habilitar el salón de conferencias "Jorge Sahade" de la FACES (figura 10) para brindar charlas cada hora a los visitantes y explicarles lo que sucedería durante el eclipse interplanetario. 


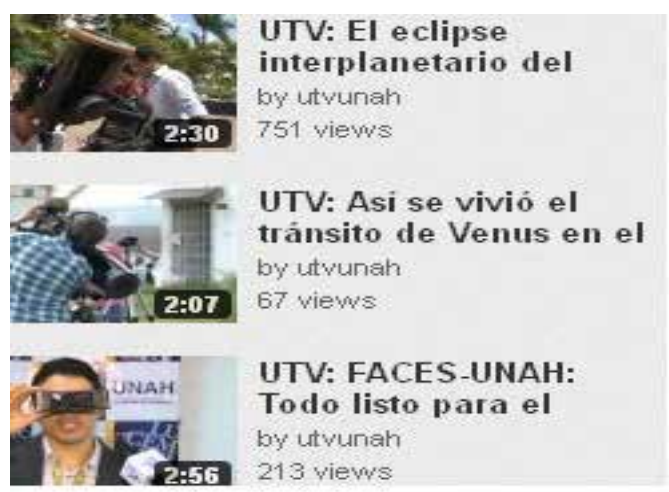

Figura 8. Videos producidos por UTV

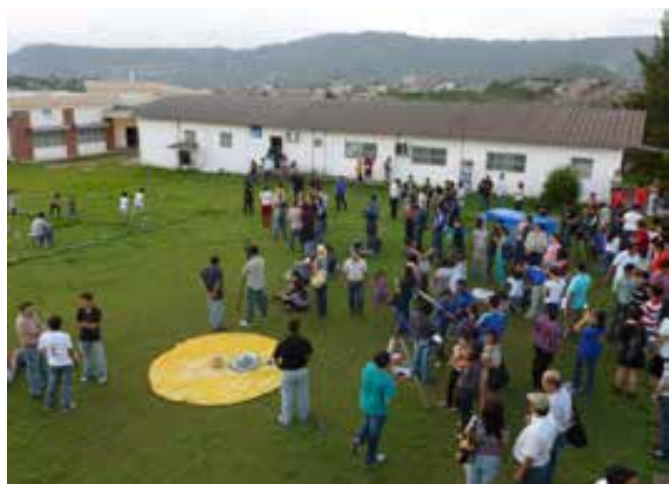

Figura 9. Parte de los visitantes al OACS el día 5 de junio de 2012. Tránsito de Venus

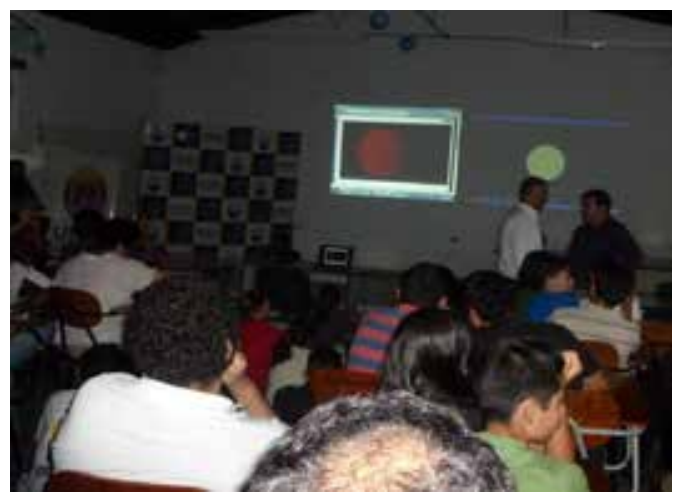

Figura 10. Salón de conferencias "Jorge Sahade" 
Se hizo un monitoreo de las posibles condiciones de clima que prevalecerían el día 5 junio en horas de la tarde. El registro obtenido indicaba cielos nublados para Tegucigalpa en la fecha y hora en que sucedería el evento, previendo esta situación la CTV tomo la decisión de conformar dos equipos de observación, uno de ellos que obtendría las imágenes desde las instalaciones del OACS, el otro lo haría desde la zona norte del país la cual presentaba mejores pronósticos para la observación astronómica. Cabe señalar que las imágenes obtenidas del Tránsito de Venus fueron captadas en la comunidad de Sambo Creek, lat. $15^{\circ} 47^{\prime} 35^{\prime \prime}$, Ion. $-87^{\circ} 22^{\prime}$ 6" ubicada en la ciudad de La Ceiba.

Gracias a la Dirección Ejecutiva de Gestión de Tecnología (DEGT) se logró contar con un ancho de banda de $10 \mathrm{Mbs}$, esto permitió que la transferencia de las imágenes así como el seguimiento vía internet de nuestra transmisión del evento tuviera el flujo de datos deseado.

\section{Conclusiones sobre la cobertura del evento}

Durante el evento se atendió un aproximado de 600 personas que visitaron el OACS y se contabilizaron 25 entrevistas ofrecidas a medios de comunicación escritos, televisivos y radiales.

Se realizaron dos programas en vivo a los noticieros: Así se informa de canal 36 y Abriendo Brecha que se transmite por los canales 10 y 7 .

Para que el público tuviera la oportunidad de observar de manera segura el Tránsito de Venus se instalaron: 2 telescopios equipados con sungun, 1 Telescopio con una pantalla de proyección y una pantalla en la cual se proyectaba la Transmisión vía internet.

Aunque no se cuenta con un registro cuantitativo del total de hondureños que obtuvieron la información brindada por el OACS y el DAAF acerca del fenómeno, una evaluación sobre la cobertura que se logró hacer a través de los medios de información por parte de la Vicerrectoría Académica de la UNAH valoró este trabajo como un éxito. 


\section{Agradecimientos}

Para el logro de esta labor fue necesario el esfuerzo de todo el personal administrativo, docente, técnico y de servicio de la FACES al cual queremos reconocerle su entrega al trabajo solicitado.

A las distintas dependencias de la UNAH que apoyaron de diferente manera la divulgación, las necesidades técnicas y administrativas requeridas para el buen resultado obtenido.

A todos los medios de comunicación masiva, escritos, radiales y televisivos. Incluyendo los medios digitales de la UNAH, como algunos de carácter privado que apoyaron con la publicidad del evento.

Al Dr. Armando Euceda por conceder un programa completo a la FACES acerca del Tránsito de Venus, en su espacio televisivo 10 al 10 que se trasmite por canal TEN.

\section{Bibliografía}

- Lu, Lingfeng, y Huifang Li. «CHINESE RECORDS OF THE 1874 TRANSIT OF VENUS.» Journal of Astronomical History and Heritage, 2013: 45-54.

- Daassou, A, Z Benkhaldoun, y Y Elazhari. «State of the art on the detection of exoplanets by the transit method with small instruments.» Laboratoire de Physique des Hautes Energies et Astrophysique, 2010.

- Smiley, Charles H. «The antiquity and Precision of Mayan Astronomy.» Journal of Royal Astronomical Society of Canada, 1960: 222.

- Banco Centroamericano de Integración Económica. «Ficha estadística de Honduras.» 2011. http://www.bcie.org/uploaded/content/article/1944368211.pdf (último acceso: sábado de mayo de 2013). 